\title{
Germplasm Acquisition from Conception to Products
}

\author{
sponsored by the
}

ASHS Genetics and Germplasm Working Group

held at the

ASHS Centennial Conference

Providence, Rhode Island

3 October 2003 